This is a post-print of the following article: Baker, K. L. \& Curtin, W. A. Assessment of phase-field-crystal concepts using long-time molecular dynamics. Physical Review B 91, (2015). The final publication is available at http://dx.doi.org/10.1103/PhysRevB.91.014103 @American Physical Society

\title{
Assessment of Phase-Field-Crystal Concepts using Long-Time Molecular Dynamics
}

\author{
K. L. Baker and W. A. Curtin \\ École Polytechnique Fédérale de Lausanne, CH-1015 Lausanne
}

(Dated: December 10, 2014)

\begin{abstract}
The ability of the phase-field-crystal (PFC) model to quantitatively predict atomistic defect structures in crystalline solids is addressed. First, general aspects of the PFC model are discussed within the context of obtaining quantitative results in solid materials. Then a specific example is used to illustrate major points. Specifically, accelerated molecular dynamics is used to compute the oneparticle probability density, $\rho^{(1)}(\boldsymbol{r})$, in a complex atomistic defect consisting of a Lomer dislocation with an equilibrium distribution of vacancies in the core, and the results are considered within the general framework of the PFC model. As expected, $\rho^{(1)}(\boldsymbol{r})$ shows numerous spatially localized peaks with integrated densities smaller than unity, as would arise in a PFC computation. However, the $\rho^{(1)}(\boldsymbol{r})$ actually corresponds to a time-averaged superposition of a few well-defined atomic configurations each having a well-defined energy. The deconvolution of $\rho^{(1)}(\boldsymbol{r})$ to obtain the actual distinct atomic configurations is not feasible. Using a potential energy functional that accurately computes the energies of distinct configurations, the potential energy computed using $\rho^{(1)}(\boldsymbol{r})$ differs from the actual average atomistic energy by $\sim 50 \mathrm{eV}$ divided among approximately 46 atoms in the core of the defect. Attempts to rectify this deviation by introducing correlations can not significantly reduce this error. The simulations show that energy barriers between distinct configurations varying by up to $0.5 \mathrm{eV}$, indicating that the simple kinetic evolution law used in PFC cannot accurately capture the true time evolution in this problem. Overall, these results demonstrate, in one non-trivial case, that the PFC model is probably unable to predict atomistic defect structures, energies, or kinetic barriers, at the quantitative levels needed for application to problems in materials science.
\end{abstract}

\section{INTRODUCTION}

There is a strong driving force in materials science to develop methods that bridge time scales from the fempto-pico-nanosecond range of direct molecular dynamics (MD) to the micro-milli-second-hours-days time scales associated with material fabrication and in-service performance. Two methods, accelerated $\mathrm{MD}^{1-4}$ and kinetic Monte $\mathrm{Carlo}^{5-7}$, are based on fundamental statistical mechanics, with well-defined approximations and sources of error, but often with high computational cost. These methods provide results of high accuracy and are faithful to the detailed atomistic mechanisms, within the limits of the interatomic potentials used in the modeling. A newer approach is the phase-field-crystal (PFC) model, which extends the continuum phase-field concept down to the atomistic scale to obtain atomic-scale resolution while following long-time evolution within a computationally feasible framework ${ }^{8}$. Current PFC formulations are approximations to the classical density functional theory (DFT) similar to those used for freezing ${ }^{9,10}$, but rely on simplifications of the theory to remain computationally efficient. While having spatial variations at the atomic scale, issues of quantitative accuracy remain and have not yet been fully assessed.

The need for atomic-scale resolution is associated with the need to achieve quantitative and predictive results of relevance for materials science. Mechanical properties of metals such as plastic flow, creep, fracture toughness, and fatigue behavior, are controlled by defects in the crystal lattice and, moreover, by the interactions among defects. The defects of greatest importance in this domain are dislocations, i.e. slip discontinuities in the crystal lat- tice, whose motion controls plastic straining, creep, and other deformation modes in metals. Dislocations have important properties at two scales. First, there are the long-range elastic fields generated by the slip discontinuity, which is characterized by the dislocation Burgers vector. Second, there are the very local atomic-scale deformations in the dislocation core that resolve the slip discontinuity over several lattice spacings. The longrange elastic interactions are accurately accounted for via elasticity theory, with no need to resolve the core structure, and this forms the basis of continuum discrete dislocation dynamics (DDD) models ${ }^{11}$. The core structure controls important material properties like the Peierls stress (stress needed to initiate motion of an initial straight dislocation $)^{12}$, some aspects of mobility as the dislocation glides ${ }^{13}$, the strongest interactions of the dislocation with other defects such as solutes ${ }^{14}$, vacancies, and grain boundaries ${ }^{15}$, and the strength of dislocation/dislocation junctions where individual cores intersect to form new atomistic structures ${ }^{16}$. As a result, huge efforts have been made over the past few decades to develop accurate interatomic potentials that are capable of properly reproducing dislocation core structures and dislocation/defect interactions ${ }^{17-22}$ to achieve the quantitative accuracy need for a fundamental understanding of the mechanical behavior of metals.

Recent work has applied the PFC method to study dislocations and their motion in face centered cubic (FCC) crystals $^{23,24}$. Because the PFC models can be tuned to achieve a desired lattice constant and elastic constants, the long-range elastic fields of a dislocation can be captured in PFC models because those fields only depend on the Burgers vector (a primitive lattice vector) and the elastic constants. However, DDD models already cap- 
ture such features. Thus, any advantage of a PFC model must lie in its ability to predict the atomistic dislocation core structure, core energy, core deformation under load, and/or core interactions with other defects. The PFC model can be designed to include individual vacancies ${ }^{25}$, so that dislocation cores in PFC include the equilibrium interaction with vacancy defects at finite temperature and long times, and this is an apparent strength of PFC relative to atomistic methods (e.g. MD). But the ability of a PFC model to accurately represent the dislocation core structure remains unexamined.

More generally, the quantitative ability of the PFC model to predict atomic-scale defects remains largely unstudied. Analyses are mainly limited to visual comparisons and relative energetics. Often, it is stated that the PFC-computed energies are not quantitatively comparable to molecular methods but that they follow similar trends as a function of some structural parameter, for instance grain boundary orientation ${ }^{26,27}$. However, to capture the structure, properties, and stress-driven thermally-activated phenomena that control kinetics requires accurate absolute energies. Mechanical phenomena are driven by applied stresses and the stored mechanical energy, which depend on the stress and the elastic moduli. Motion, nucleation, and/or interaction of defects depends on the core energy changes in these processes relative to the available stored mechanical energy. Absolute energy differences are thus essential. Poorly predicted core energies will lead to incorrect conclusions about defect properties, even if the stored mechanical energy is predicted accurately. Therefore, PFC must be quantitatively accurate at all levels.

With the above remarks as background, in this paper we report progress toward assessment of the general PFC framework to quantitatively predict atomistic structure and energetics of a non-trivial atomistic defect in a crystalline material. We choose a basic problem, the structure of a dislocation core at thermal equilibrium including vacancies in the core, and analyze the actual time-average atomic structure, individual configurations, potential energy, and ideal free energy emerging from long-time MD. We then examine (i) how this timeaveraged structure could be interpreted within a PFC framework to yield understanding of the actual underlying defect, (ii) whether PFC free energy models can predict the energies of the defect, and (iii) whether PFC can capture the kinetic processes occurring within the dislocation core. We find, in general, that a PFC framework presents difficulties in all of the above aspects, suggesting that PFC-type formulations are unable to provide the quantitative understanding that is required for predictive, mechanistic materials science modeling of defects in crystals.

The rest of this paper is organized as follows. Section
II contains a brief review of classical Density Functional Theory and the approximations leading to PFC-type theories. In Section III, the test system of a dislocation core in aluminum is introduced and the detailed application of accelerated molecular dynamics to calculate the one-particle density and average potential energy of the system is described. In Section IV, the MD results and their interpretation with regard to structure, energetics, and kinetics, within the context of a PFC analysis is presented. Section V provides some concluding remarks.

\section{THE PHASE-FIELD-CRYSTAL METHOD}

\section{A. PFC from Classical Density Functional Theory}

The formal justification for the PFC framework rests on a theorem of classical density functional theory stating that the equilibrium Helmholtz free energy, $F$, of a system of $N$ atoms is a unique functional of the one-particle atomic density ${ }^{28,29}$. The one-particle density, $\rho^{(1)}(\boldsymbol{r})$, is defined as the ensemble average of the one-particle density operator ${ }^{28,29}$

$$
\rho^{(1)}(\boldsymbol{r})=\left\langle\sum_{i=1}^{N} \delta\left(\boldsymbol{r}-\boldsymbol{r}_{\boldsymbol{i}}\right)\right\rangle,
$$

where the delta functions specify the positions $\boldsymbol{r}_{\boldsymbol{i}}$ of each of the $N$ atoms in the system at any instant of time, and the average is over time or ensembles. The total Helmholtz free energy functional of the one-particle density can be divided into ideal, excess, and external parts $\operatorname{as}^{28,29}$

$$
F\left[\rho^{(1)}\right]=F^{i d}\left[\rho^{(1)}\right]+F^{e x}\left[\rho^{(1)}\right]+F^{e x t}\left[\rho^{(1)}\right],
$$

where

$$
F^{i d}\left[\rho^{(1)}\right]=\beta^{-1} \int \rho^{(1)}(\boldsymbol{r})\left(\ln \left(\rho^{(1)}(\boldsymbol{r}) \Lambda^{3}\right)-1\right) d \boldsymbol{r},
$$

and

$$
F^{e x t}\left[\rho^{(1)}\right]=\int \rho^{(1)}(\boldsymbol{r}) V^{e x t}(\boldsymbol{r}) d \boldsymbol{r} .
$$

where $\beta=\frac{1}{k_{B} T}, k_{B}$ is Boltzmann's constant, $T$ is the temperature, $\Lambda$ is the thermal de Broglie wavelength, and $V^{e x t}(\boldsymbol{r})$ is any imposed external potential. The excess free energy, $F^{e x}$, is unknown in general. The first step in approximating $F^{e x}$ using DFT, proposed for modeling freezing transitions ${ }^{9,10}$, is a functional expansion about a uniform reference liquid of density $\rho_{0}$ truncated to second order $^{28}$, 


$$
F^{e x}\left[\rho^{(1)}\right]=F_{L}^{e x}-k_{B} T \int c_{L}^{(1)} \Delta \rho^{(1)}\left(\boldsymbol{r}_{\mathbf{1}}\right) d \boldsymbol{r}_{\mathbf{1}}-\frac{k_{B} T}{2} \int \Delta \rho^{(1)}\left(\boldsymbol{r}_{\mathbf{1}}\right) \Delta \rho^{(1)}\left(\boldsymbol{r}_{\mathbf{2}}\right) c_{L}^{(2)}\left(r_{12}\right) d \boldsymbol{r}_{\mathbf{1}} d \boldsymbol{r}_{\mathbf{2}},
$$

where $\Delta \rho^{(1)}(\boldsymbol{r})=\rho^{(1)}(\boldsymbol{r})-\rho_{0}, F_{L}^{e x}$ is the excess Helmholtz free energy of the reference liquid, $r_{12}=\left|\boldsymbol{r}_{\mathbf{1}}-\boldsymbol{r}_{\mathbf{2}}\right|$, and $c_{L}^{(1)}$ and $c_{L}^{(2)}$ are the first two liquid direct correlation functions defined $\operatorname{as}^{28,29} c_{L}^{(n)}\left(\boldsymbol{r}_{\mathbf{1}}, \ldots, \boldsymbol{r}_{\boldsymbol{n}}\right)=-\beta \frac{\delta F_{L}^{e x^{n}}\left[\rho^{(1)}\right]}{\delta \rho^{(1)}\left(\boldsymbol{r}_{\mathbf{1}}\right) \ldots \delta \rho^{(1)}\left(\boldsymbol{r}_{\boldsymbol{n}}\right)}$. Since $c_{L}^{(1)}$ is related to the chemical potential $\mu_{L}$ and the integral of $c_{L}^{(2)}$ is related to the liquid compressibility, it is useful to rewrite Eq. 5 in terms of thermodynamic variables as

$$
F^{e x}\left[\rho^{(1)}\right]=F_{L}^{e x}+\mu_{L}\left(N-N_{0}\right)-\left(\left.\frac{V^{2}}{N_{0}} \frac{\partial P}{\partial V}\right|_{e x}\right)\left(N-N_{0} / 2\right)-\frac{k_{B} T}{2} \int \rho^{(1)}\left(\boldsymbol{r}_{\mathbf{1}}\right) \rho^{(1)}\left(\boldsymbol{r}_{\mathbf{2}}\right) c_{L}^{(2)}\left(r_{12}\right) d \boldsymbol{r}_{\mathbf{1}} d \boldsymbol{r}_{\mathbf{2}},
$$

where $N$ and $N_{0}$ are the total number of atoms in the solid and reference system, respectively, $V$ is the system volume, $P$ is the pressure, and $d P /\left.d V\right|_{e x}$ is the excess compressibility above the ideal-gas value. It is now clear that only the fourth term in Eq. 6 depends on the atomic structure $\rho^{(1)}$. Therefore, minimizing the total free energy with respect to $\rho^{(1)}$ is reduced to a competition between the ideal free energy, any contribution from the external potential, and only the last term in Eq. 6 .

The DFT theory introduced above strictly applies only to the ground state. Therefore, it might seem unable to predict the one-particle density or free energy of any metastable states. However, this is where the introduction of a $V^{e x t}$ is useful. A hypothetical $V^{e x t}$ can be introduced to constrain the system to lie within the basin of any metastable state. If computation of the metastable state free energy yields a density $\rho^{(1)}(\boldsymbol{r})$ that is localized near to the minimum energy of the metastable basin, then the presence of $V^{e x t}$ has no practical effect on either the density or resulting free energy. Thus, DFT can be used to obtain the Helmholtz free energy for such metastable states. An external potential is also often used, explicitly or implicitly, to enforce boundary conditions on a DFT model, such as constraining the system against rigid body rotations or translations, or imposing a density at the boundaries of a simulation cell.

Existing PFC models use the equilibrium Helmholtz free energy functional described above but with further approximations to make computations less demanding. In solid/crystalline materials, minimization of the free energy using Eq. 6 leads to density profiles consisting of very localized peaks, corresponding to thermal vibrations of atoms around equilibrium sites, but the computational efficiency of PFC decreases dramatically as sharper density peaks are considered ${ }^{23}$. Therefore, most PFC formulations also (i) expand $F^{i d}$ in a power series; (ii) restrict $\rho^{(1)}(\boldsymbol{r})$ to a fourier-space representation limited to one or two families of Fourier coefficients/reciprocal lattice vectors of the underlying crystal structure, and (iii) use $c_{L}^{(2)}$ as a fitting function to achieve some desired material properties. These simplifications make free energy computations much more efficient, but cause some conceptual and quantitative problems as described later. Additional ad-hoc terms can be added to the free energy functional to accomplish other goals, like enforcing only positive density to allow vacancy structures to be stable ${ }^{25}$. It is these simplified or augmented models that have been used to predict defect structures (dislocations ${ }^{23,24,30-32}$, grain boundaries ${ }^{26,27,33-35}$, crystal-liquid interfaces ${ }^{26,36-41}$, etc.).

\section{B. An Alternative Functional}

There exists another class of free energy functionals that take a different approach. Instead of expanding about a liquid (high-temperature) reference, one can expand around the zero-temperature solid. That is, the Helmholtz free energy functional is developed within the harmonic theory of small amplitude vibrations around the equilibrium atomic positions (phonon theory in perfect crystals), which is highly accurate even close to the melting point ${ }^{42}$. The equilibrium Helmholtz free energy can be written in terms of the internal energy $E$ and entropy $S$ as

$$
F\left[\rho^{(1)}\right]=E\left[\rho^{(1)}\right]-T S\left[\rho^{(1)}\right]+F^{e x t} .
$$

The entropy and internal energy can be divided into ideal and excess parts where $E^{i d}$ is the kinetic energy, $E^{e x}=$ $U$ is the potential energy, $S^{i d}$ is the ideal gas entropy, and $S^{e x}$ is the excess entropy arising from inter-particle interactions. The free energy is then

$$
F\left[\rho^{(1)}\right]=F^{i d}\left[\rho^{(1)}\right]+U\left[\rho^{(1)}\right]-T S^{e x}\left[\rho^{(1)}\right]+F^{e x t} .
$$

where $F^{i d}=E^{i d}-T S^{i d}$ was defined previously. The potential energy functional $U$ can be written $\operatorname{as}^{28}$ 
This is a post-print of the following article: Baker, K. L. \& Curtin, W. A. Assessment of phase-field-crystal concepts using long-time molecular dynamics. Physical Review B 91, (2015). The final publication is available at http://dx.doi.org/10.1103/PhysRevB.91.014103 @American Physical Society

$$
U\left[\rho^{(1)}\right]=\int\left(\prod_{i=1}^{N} \rho^{(1)}\left(\boldsymbol{r}_{\boldsymbol{i}}\right)\right) g^{(N)}\left(\boldsymbol{r}_{\mathbf{1}}, \ldots, \boldsymbol{r}_{\boldsymbol{N}}\right) \phi^{(N)}\left(\boldsymbol{r}_{\mathbf{1}}, \ldots, \boldsymbol{r}_{\boldsymbol{N}}\right) d \boldsymbol{r}_{\mathbf{1}} \ldots d \boldsymbol{r}_{\boldsymbol{N}}
$$

where $g^{(n)}\left(\boldsymbol{r}_{\mathbf{1}}, \ldots, \boldsymbol{r}_{\boldsymbol{n}}\right)$ is the $n$-particle correlation function of the solid, and $\phi^{(n)}\left(\boldsymbol{r}_{\mathbf{1}}, \ldots, \boldsymbol{r}_{\boldsymbol{n}}\right)$ is the $n$-body potential energy function. In the case of a pair potential, $\phi^{(2)}\left(r_{12}\right)$, equation 9 reduces to 28

$$
U\left[\rho^{(1)}\right]=\frac{1}{2} \int \rho^{(1)}\left(\boldsymbol{r}_{\mathbf{1}}\right) \rho^{(1)}\left(\boldsymbol{r}_{\mathbf{2}}\right) g^{(2)}\left(\boldsymbol{r}_{\mathbf{1}}, \boldsymbol{r}_{\mathbf{2}}\right) \phi^{(2)}\left(r_{12}\right) d \boldsymbol{r}_{\mathbf{1}} d \boldsymbol{r}_{\mathbf{2}}
$$

where $r_{12}=\left|\boldsymbol{r}_{\mathbf{1}}-\boldsymbol{r}_{\mathbf{2}}\right|$. From this exact result, the excess entropy in equation 8 is then neglected as small (it is zero at $\mathrm{T}=0$ ) or is included via a self-consistent harmonic theory. The free energy is thus approximated as $F=F_{i d}+U+F^{e x t}$. The pair correlation of the solid remains, and must be approximated. However, the pair correlation function $g^{(2)}$ is relatively featureless. The pair distribution function for the solid, $\rho^{(2)}=$ $\rho^{(1)}\left(\boldsymbol{r}_{\mathbf{1}}\right) \rho^{(1)}\left(\boldsymbol{r}_{\mathbf{2}}\right) g^{(2)}\left(\boldsymbol{r}_{\mathbf{1}}, \boldsymbol{r}_{\mathbf{2}}\right)$, is dominated by the spatial variations in $\rho^{(1)}$, and the main feature of $g^{(2)}$ is a coreexclusion to avoid double-counting so that the sum rule $\int \rho^{(1)}\left(\boldsymbol{r}^{\prime}\right) g^{(2)}\left(\boldsymbol{r}, \boldsymbol{r}^{\prime}\right) d \boldsymbol{r}^{\prime}=N-1$ is satisfied.

We now compare the DFT form for $F$ using Eq. 6 to the harmonic-solid form using Eqs. 8 and 10 for a pairpotential system. Aside from a constant term, the functional forms are identical, differing only in the function multiplying the one-particle densities within the double integral, with $k_{B} T c_{L}^{(2)}$ appearing in the PFC functional and $\phi^{(2)} g^{(2)}$ appearing in the harmonic theory. Since $c_{L}^{(2)} \sim \phi^{(2)} / k_{B} T$ and $g^{(2)} \sim 1.0$ for large pair separations $r_{12}$, these two terms are equivalent at large $r_{12}$. However, at short distances, there are significant differences and the PFC model only contains an additional set of constant terms to attempt to correct for any differences between Eq. 6, which is based on an expansion, and Eq. 10, which is based on the assumption of small vibrations. In addition, as will be shown later, the potential energy functional for $U$ can be easily extended to multi-body potential interactions, while Eq. 6 remains a pair-interaction model, hence limiting the quantitative description of many materials, such as metals, where such multi-body interactions are essential.

\section{General Comments on the PFC Model}

With the above formal background, we now discuss some general aspects of the PFC method. The PFC method is often considered as an extension of the mesoscale phase-field method. In the standard mesoscale phase-field models, the interface width representing the interface between phases is negligible compared to other important physical dimensions and the theory can be shown to asymptotically approach a sharp interface model ${ }^{43}$; the width of the interface then becomes relatively unimportant and can be chosen for computational efficiency. In PFC, the phase field varies at the atomic scale; the "width" of the phase-field interface is intrinsic to the problem - it reflects the vibrational amplitude of the atoms - and it intrinsically influences the predicted structure and free energy. Therefore, when the "interface" is smeared by not fully resolving the sharp density field $\rho^{(1)}$, there is no separation of spatial scales. This leads to an intimate mixing of physical contributions to the free energy and computational approximations made for numerical efficiency.

A common outcome of PFC computations is the existence of defect structures wherein the integrated number of atoms within one density peak is smaller than unity, i.e. less than one atom (e.g. ${ }^{8,26,27,34,36,44-46}$ ). Such structures are to be expected in solids where diffusion is present, and simply mean that, on average, an atom is around that location for only some fraction of the time. The interpretation of the defect structure is, however, complicated. Using a liquid-like $c_{L}^{(2)}$, and thus liquid like $g_{L}^{(2)}$, violations of the sum rule $\int \rho^{(1)}\left(\boldsymbol{r}^{\prime}\right) g^{(2)}\left(\boldsymbol{r}, \boldsymbol{r}^{\prime}\right) d \boldsymbol{r}^{\prime}=$ $N-1$ are inevitable, and hence the computed free energy using Eq. 6 is unlikely to be accurate.

As stated in the PFC literature, there is a poor correspondence between PFC and DFT even when the PFC parameters for the functional expansions are fit to the DFT energy functional ${ }^{26,47}$. Improved results can be achieved when a more accurate fit of the first peak of the $c_{L}^{(2)}$ function is used ${ }^{26}$ and polynomial functions can be used to fit several peaks of $c_{L}^{(2)}$ and bring results very close to the original DFT ${ }^{41}$. Still, the power series expansion of $F^{i d}$ is valid only for a slowly varying $\rho^{(1)}$, yet is always used for numerical efficiency and can lead to discontinuous density fields if $c_{L}^{(2)}\left(r_{12}\right)$ goes to zero at large $r_{12}^{41}$.

Beyond any approximation that PFC makes, the accuracy of the DFT functional expansion of the free energy about the liquid state limits the accuracy of the PFC method. While formally exact, this expansion of the free energy about a liquid state cannot be shown to converge to the exact excess free energy as more terms of the expansion are used ${ }^{48}$. In fact, it was shown that using a truncated expansion to second order is only fortuitously accurate for predicting freezing transitions, and adding third order terms caused dramatic deviations from the expected results ${ }^{49}$. In contrast, the alternative DFT functional based on Eq. 8 can accurately predict thermo- 
This is a post-print of the following article: Baker, K. L. \& Curtin, W. A. Assessment of phase-field-crystal concepts using long-time molecular dynamics. Physical Review B 91, (2015). The final publication is available at http://dx.doi.org/10.1103/PhysRevB.91.014103 @American Physical Society

dynamic properties of crystals and defects in the absence of atomic diffusion ${ }^{50}$.

There is the hope that by fitting aspects of the PFC free energy functional, PFC could be become quantitatively accurate for predicting specific properties. This procedure is akin to fitting empirical interatomic potentials to specific properties, but the tradeoffs in accuracy between different properties seems more severe. For example, stacking faults can be stabilized in an FCC crystal by using a simplified $c_{L}^{(2)}$ that artificially lowers the stacking fault energy, but this approximation limits the stability of the FCC phase itself ${ }^{23}$. By artificially biasing the free energy, stacking faults become more stable, but the range of possible FCC crystal elastic constants becomes limited ${ }^{23}$. In contrast, interatomic potentials are developed to simultaneously capture many properties, both bulk and defect, of the material with high accuracy, and the potentials have significant flexibility to minimize trade-offs. Furthermore, such potentials are extremely material-specific, and can then be used as input to a more-general functional (e.g. Eq. 10) rather than having the functional and the material properties closely linked as in PFC.

Another important aspect of the PFC method is that the PFC functional is supplemented by an evolution law to capture kinetic processes at time scales much longer than are accessible by MD. Specifcially, PFC methods evolve a dimensionless density $\psi=\rho^{(1)} / \rho_{0}-1$ using a kinetic law of the form

$$
\frac{\delta \psi}{\delta t}=A^{2} \nabla^{2} \frac{\delta F[\psi]}{\delta \psi}
$$

where the constant $A$ controls the diffusive mobility ${ }^{8}$. Newer PFC formulations often use a modified version of Eq. 11

$$
\frac{\delta^{2} \psi}{\delta t^{2}}+B \frac{\delta \psi}{\delta t}=A^{2} \nabla^{2} \frac{\delta F[\psi]}{\delta \psi}
$$

were the constant $B$ controls the elastic relaxation rate ${ }^{30}$. These dynamical equations originate from the macroscopic phase field method, and drive the system to lower free energy by moving downhill via gradients of the free energy functional. However, there is no corresponding time-dependent DFT theory that underpins this kinetic assumption. Time-dependent DFT theories refer to problems in which the external potential $V^{e x t}$ is an explicit function of time ${ }^{51}$. Furthermore, the PFC dynamics with a constant diffusive mobility does not take into consideration the specific state-to-state rare-event transitions via thermal fluctuations over energy barriers that are known to control the kinetic evolution of solids.

In addition, since DFT methods only apply to equilibrium, the introduction of dynamics inappropriately mixes timescales. At any instant of time, the DFT functional is used to compute a free energy that corresponds to a long-time average of the system in its current state, even though the system is not in equilibrium and is changing with time. Implicit also is the assumption that the interatomic correlations in equilibrium also apply in nonequilibrium, and that the diffusive mobility is a constant everywhere (see Emmerich et al. $2012^{52}$ and references therein).

In the following sections, we use long-time, accelerated MD simulations of a non-trivial atomistic defect to compute $\rho^{(1)}(\boldsymbol{r})$, and compute the energy from this density, to highlight some of the issues noted above in a specific and quantitative case of interest in materials science.

\section{HYPERDYNAMICS SIMULATION OF A LOMER DISLOCATION IN EQUILIBRIUM WITH VACANCIES}

We study a Lomer dislocation in Aluminum with a near-equilibrium vacancy concentration in the core. The Lomer dislocation is created within an atomistic simulation cell by applying the elastic solution for the dislocation displacement field to a perfect crystal aligned such that $x=[\overline{1} 10], y=[001], z=[110]$ with periodicity along the dislocation line (the $z$ direction), a Burgers vector $\boldsymbol{b}=\frac{a}{2}[\overline{1} 10]$, and lattice constant $a$ at $\mathrm{T}=300 \mathrm{~K}$. All atoms within a cylinder of radius $10 \AA$ and length $28.6 \AA$ around and along the dislocation line (612 atoms in total) are allowed to relax with all outer atoms held fixed. We use the Ercolessi-Adams embedded-atom potential for $\mathrm{Al}^{19}$ and the LAMMPS ${ }^{53}$ MD simulation package with a Langevin thermostat. Using the $T=0 \mathrm{~K}$ structure, we first measure the vacancy formation energy in sites around the core. There are only two sets of equivalent sites where the vacancy formation energy $(0.044 \mathrm{eV})$ is reduced significantly relative to the bulk value $(0.69$ $\mathrm{eV}^{54}$ ). In thermodynamic equilibrium at $\mathrm{T}=300 \mathrm{~K}$, these two types of atomic sites have an equilibrium vacancy concentration of $\sim 9$ percent $^{54}$. Therefore, two vacancies are added (i.e. two atoms are deleted) in our simulation cell in two of these sites to create a vacancy concentration of $1 / 12 \approx 8.3 \%$ in those sites. This configuration thus already accounts for the long-range vacancy diffusion needed to reach this near-equilibrium state (which would have occurred by vacancy diffusion with a migration enthalpy in bulk $\mathrm{Al}$ of $0.61 \mathrm{eV}^{54}$ ). Starting from one initial configuration of the vacancies (Figure 1a), we run hyperdynamics ${ }^{1,2}$ simulations using the simplified bond-boost method ${ }^{55}$ to evolve the system through the accessible phase space at $\mathrm{T}=300 \mathrm{~K}$ over times sufficient to reach a near-equilibrium statistical sampling. We use a conservative value of 0.2 for the maximum bond strain $\left(\epsilon_{\alpha}\right.$ from $\left.^{55}\right)$, and a maximum boost-energy of $0.2 \mathrm{eV}\left(S_{\alpha}\right.$ from $^{55}$ ) to maintain efficient sampling of the boosted potential yet provide reduced transition times to configurations inaccessible using standard MD.

We compute the one-particle density, $\rho^{(1)}(\boldsymbol{r})$, by performing a time average during the accelerated MD simulation according to Equation 1 as follows. In any given 
This is a post-print of the following article: Baker, K. L. \& Curtin, W. A. Assessment of phase-field-crystal concepts using long-time molecular dynamics. Physical Review B 91, (2015). The final publication is available at http://dx.doi.org/10.1103/PhysRevB.91.014103 @American Physical Society

low energy configuration (i.e. a local energy minimum with thermal vibrations), the positions of the atoms are recorded for 10 picoseconds on a grid of $0.06 \times 0.06 \times$ $0.07 \AA$ in the $\mathrm{x}, \mathrm{y}$, and $\mathrm{z}$ directions to obtain the oneparticle density for that configuration. The bond-boost potential is then applied to accelerate a transition into a new configuration. The real time spent in the previous configuration is computed using the standard hyperdynamics procedure. The one-particle density is then calculated by averaging over all of the one-particle densities in each configuration weighted by the real time spent in each configuration,

$$
\rho^{(1)}(\boldsymbol{r})=\frac{\sum_{i=1}^{N_{\text {states }}} t_{i} \rho_{i}^{(1)}(\boldsymbol{r})}{\sum_{i=1}^{N_{\text {states }}} t_{i}},
$$

where $N_{\text {states }}$ is the total number of configurations observed, $\rho_{i}^{(1)}(\boldsymbol{r})$ is the one-particle density from configuration $i$, and $t_{i}$ is the time spent in configuration $i .1448$ low energy configurations (many being similar aside from periodic translations) are observed during the simulation so that the one-particle density calculated here is close to the final equilibrium density. We note that configurations in which the vacancies move out of the immediate core region are at least $0.447 \mathrm{eV}$ higher in energy than the vacancies in their lowest energy configurations, and thus are approximately $3.1 \times 10^{-8}$ times less likely to occur; inadequate sampling of those configurations thus has a negligible effect on the one-particle density.

Similarly, we compute the potential energy of the MD system as the time-weighted average of the potential energies of the low energy configurations in the same manner as the time-weighted one-particle density,

$$
U=\frac{\sum_{i=1}^{N_{\text {states }}} t_{i} U_{i}}{\sum_{i=1}^{N_{\text {states }}} t_{i}},
$$

where $U_{i}$ is the total potential energy of each minimum.

\section{RESULTS AND DISCUSSION}

We frame our discussion around three aspects of the PFC method: predicted structure and its interpretation, energies of the structure, and kinetics.

\section{A. Defect Structure}

During the simulation, core atoms near the vacancies diffuse within the core region and do not remain on the original lattice sites. Instead, an atom will occupy an off-lattice site between two rows of atoms that make up the dislocation core. The two vacancies start the simulation next to each other, but can separate because the double-vacancy complex is only slightly more energetically favorable $(0.04 \mathrm{eV}$ at $\mathrm{T}=0 \mathrm{~K})$ than two isolated

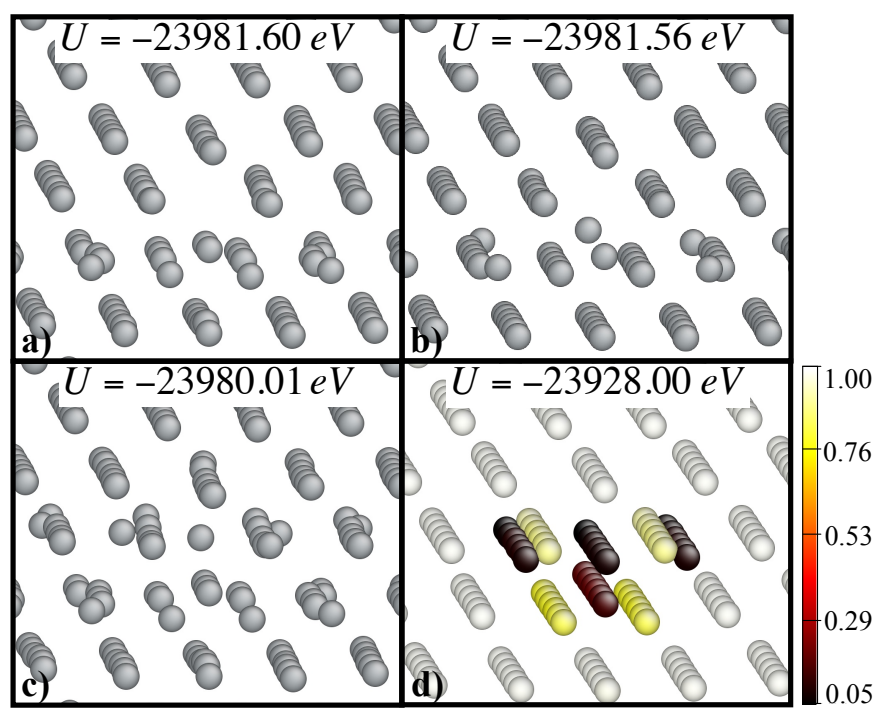

FIG. 1: (Color online) (a-c) Actual atomistic configurations of a Lomer dislocation with two vacancies that are frequently observed during accelerated MD simulations, and their associated total potential energies at $300 \mathrm{~K}$ : a) a low energy structure where vacancies have merged in the dislocation core; $b$ ) a structure where two vacancies are separated along the core; c) a structure where a third lattice vacancy is

created next to the initial two vacancies with a corresponding interstitial atom generated in the center of the core. d) The quenched one-particle density (see text) corresponding to the time-averaged one-particle density from the accelerated MD simulation, with the integrated density of each peak indicated by the color. This quenched density is used to compute the "PFC" potential energy quoted, which differs significantly from the potential energies of any individual configuration such as those shown in (a-c).

single-vacancy complexes. Since the energy difference is small, the vacancies separate and rejoin several times during the simulation. The system configuration primarily divides its time among a distinct set of specific configurations, each of which corresponds to a local free energy minimum separated from the other local configurations by some free energy barrier. Three frequently-occurring configurations (each configuration has many equivalent configurations corresponding to translations by a multiple of the lattice constant) are shown in Figures 1a-c, along with the average total potential energy of the system at $\mathrm{T}=300 \mathrm{~K}$ in each configuration.

Figures $2 \mathrm{a}$ and $\mathrm{b}$ show the one-particle density around the dislocation core. Being a time-weighted average of a set of distinct atomic configurations, the one-particle density shows a set of sharp peaks at distinct positions, corresponding to atoms that are vibrating around those positions for some time period. The one-particle density shows all possible positions of individual atoms simulta- 
This is a post-print of the following article: Baker, K. L. \& Curtin, W. A. Assessment of phase-field-crystal concepts using long-time molecular dynamics. Physical Review B 91, (2015). The final publication is available at http://dx.doi.org/10.1103/PhysRevB.91.014103 @American Physical Society

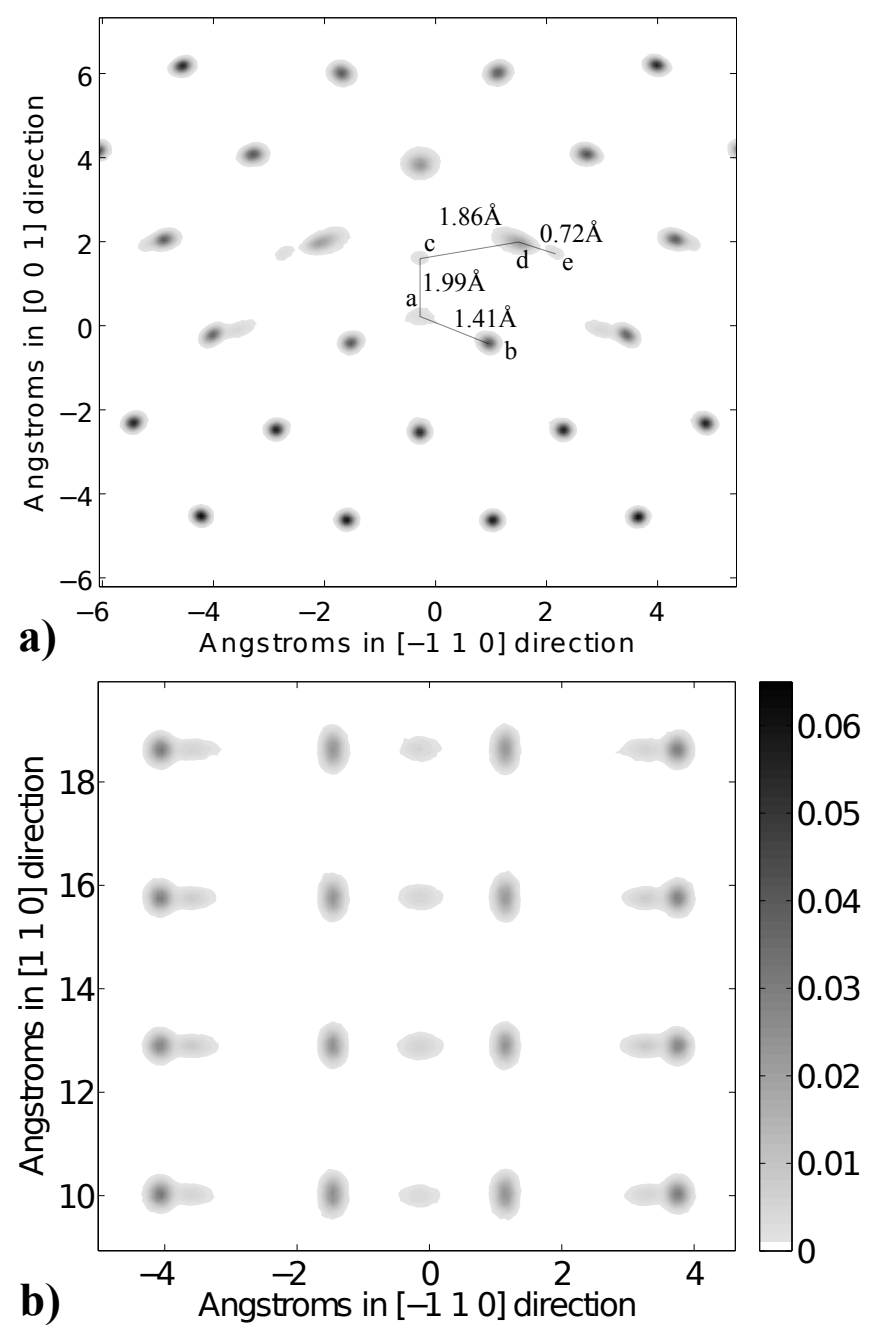

FIG. 2: One-particle density a) integrated along the $\mathrm{z}=[110]$ dislocation line direction from $z=2.4$ to $5 \stackrel{\AA}{A}$; this integrated projection corresponds to a slice perpendicular to the dislocation line containing two planes of atoms. Some density peaks in the core and their distances are labeled. b) The density integrated along the $\mathrm{y}=[001]$ direction from $y=-1$ to $1 \stackrel{\AA}{A}$; this integrated projection corresponds to a one-atom-wide slice along a plane intersecting the dislocation core.

neously without regard to spatial and temporal time correlations. The integrated density under any one sharp peak represents the fraction of time, or probability, that the region is actually occupied by an atom during the course of the simulation. Figures $2 \mathrm{a}$ and $\mathrm{b}$ are essentially the outcome of a perfect PFC calculation, i.e. they correspond to the actual $\rho^{(1)}(\boldsymbol{r})$ of a physical problem obtained by full molecular simulation with essentially no approximation. Below we analyze this result within the context of a generic PFC model.

To reduce the amount of information, we compress all of the sharp peaks in Figure 2 into a collection of
$N_{p}$ "quenched peaks" by integrating the mass density around each peak. This yields a "quenched" density $\rho_{q}^{(1)}(\boldsymbol{r})=\sum_{i=1}^{N_{p}} \rho_{i} \delta\left(\boldsymbol{r}-\boldsymbol{r}_{\boldsymbol{i}}\right)$, where $\rho_{i}$ is the integrated weight of the peak at position $\boldsymbol{r}_{\boldsymbol{i}}$ located at the centroid of the integrated density of each quenched peak. In this work, the integrated density includes all bins with nonzero density that are connected to a nearby peak, including some connected double-peaks visible in Figure 2. Figure 1d shows the "PFC" configuration of these peaks colored by their integrated mass, $\rho_{i}$, which is equal to the probability of occupation of that (quenched) position. Figure 1d is essentially the time average over all of the individual configurations similar to those shown in Figures 1a-c. Of relevance to many questions about material behavior are the individual configurations. The reconstruction of the distinct set of actual atomistic configurations (e.g. Figure 1a,b,c) using only $\rho_{q}^{(1)}(\boldsymbol{r})$ in Figure 1d seems highly non-unique and under-determined. Any such reconstruction would certainly require the use of postulated solid-structure multi-particle correlation functions that are not present within the PFC method itself. That is, if one knew the multiparticle correlation functions, one could construct possible configurations and then attempt to determine which configurations actually occurred and the time-averaged weight for each configuration. However, this is highly speculative and no tools currently exist to execute such a strategy. Thus, the PFC calculation itself does not provide direct information about the actual obtainable defect structures, i.e. the structures that control the behavior of this defect.

\section{B. Defect Energies}

We now use our "exact" PFC result for the one-particle density to attempt to answer questions of interest in materials science related to defect energies. To do this, we compute the energy of the PFC structure from the measured quenched density, $\rho_{q}^{(1)}(\boldsymbol{r})$, and compare it to the actual energies measured in the MD simulation. Since the peaks are sharp, the "quenched" density is a good approximation to the true one-particle density (as demonstrated below). Furthermore, to reduce variations in the true one-particle density along the periodic $\mathrm{z}$ direction, we average the one-particle density over the periodic spacing. This averaging removes small variations from under-sampling the rarest states, and produces a density field as close to the true equilibrium density field as possible.

Computation of the potential energy requires a potential energy functional. We will use the alternative function of Eq. 9, adapted for the many-body embedded atom potential used here along the lines demonstrated by LeSar et $a l .{ }^{56}$. The EAM potential energy includes a contribution from a pair potential and from a per-atom embedding energy $\mathcal{F}_{i}$ that is a function of the electron density at the position of atom $\boldsymbol{r}_{\boldsymbol{i}}$ due to surrounding atoms at positions $\boldsymbol{r}_{\boldsymbol{j}}$. The pair term directly fits the 
This is a post-print of the following article: Baker, K. L. \& Curtin, W. A. Assessment of phase-field-crystal concepts using long-time molecular dynamics. Physical Review B 91, (2015). The final publication is available at http://dx.doi.org/10.1103/PhysRevB.91.014103 @American Physical Society

form of Eq. 10. LeSar et al. derived an accurate approximation to the embedding term ${ }^{56}$, which has also recently been used in the PFC-like "diffusive molecular dynamics" model of Li et al. ${ }^{57,58}$. We use the formulation of LeSar et al. applied to our quenched density but extend it to include pair correlations in the calculation of the electron density of atom $i$, yielding the potential energy function

$$
\begin{gathered}
U_{D F T}=\frac{1}{2} \sum_{i=1}^{N_{p}} \sum_{j \neq i}^{N_{p}} \rho_{i} \rho_{j} g^{(2)}\left(\boldsymbol{r}_{\boldsymbol{i}}, \boldsymbol{r}_{\boldsymbol{j}}\right) \phi^{(2)}\left(r_{i j}\right) \\
+\sum_{i=1}^{N_{p}} \rho_{i} \mathcal{F}_{i}\left[\sum_{j \neq i}^{N_{p}} \rho_{j} g^{(2)}\left(\boldsymbol{r}_{\boldsymbol{i}}, \boldsymbol{r}_{\boldsymbol{j}}\right) \psi^{(2)}\left(r_{i j}\right)\right]
\end{gathered}
$$

where $r_{i j}=\left|\boldsymbol{r}_{\boldsymbol{i}}-\boldsymbol{r}_{\boldsymbol{j}}\right|$, and $\psi^{(2)}$ is the two-body electron density function ${ }^{59}$. Since all thermal vibrations have been removed from the quenched density, to compare potential energy values computed with this functional to $\mathrm{MD}$, we add the equipartition thermal potential energy of $1.5 \mathrm{~N} k_{B} \mathrm{~T}=23.65 \mathrm{eV}$ to the values computed with equation 15 to obtain the $\mathrm{T}=300 \mathrm{~K}$ potential energy.

To test the validity of our functional, we first apply it to some reference geometries and compare the predictions to the results of MD. Specifically, we compute via MD the one-particle density and average potential energy for (i) a perfect crystal and (ii) a Lomer dislocation core without vacancies. The perfect crystal is created with the same crystal orientation, fixed atom boundary beyond a circle of radius of $10 \AA$ in the $x-y$ plane, and periodic boundary conditions and thickness along the $\mathrm{z}$ direction. The Lomer dislocation core without vacancies is created in an identical way to the dislocation core with vacancies, described earlier, but without removing any atoms. The time-averaged potential energy during a normal MD simulation is computed; accelerated MD is not needed because there is no diffusion. The simulated density $\rho^{(1)}(\boldsymbol{r})$ is measured using bins and condensed into a set of $N_{p}$ quenched peaks as described above. The quenched density is used to compute the potential energy using Eq. 15. The correlation function is approximated as $g^{(2)}\left(\boldsymbol{r}_{\boldsymbol{i}}, \boldsymbol{r}_{\boldsymbol{j}}\right)=1-\delta_{i j}$, which eliminates the self-interaction and eliminates the need for the restriction $j \neq i$.

For the perfect crystal, the absolute difference between the potential energy computed with the functional of Eq. 15 and with $\mathrm{MD}$ is only $0.80 \mathrm{eV}$, or $0.0012 \mathrm{eV}$ per atom on average across the 660 atoms in the MD simulation. Atoms away from the outer boundary have an error around $0.002 \mathrm{eV}$ per atom. For the Lomer core without vacancies, the total difference between the DFT functional and $\mathrm{MD}$ is only $1.15 \mathrm{eV}$, or $0.0019 \mathrm{eV}$ per atom on average across $612 \mathrm{MD}$ atoms. Examining specific atoms in the core, two rows of atoms along the core have an error of $0.03 \mathrm{eV}$ per atom, while near the core the error ranges from -0.011 to $0.019 \mathrm{eV}$ per atom; thus there are some canceling errors when considering the total potential energy. Overall, however, the proposed $U_{D F T}$ agrees fairly well with the full MD simulations.

We now calculate $U_{D F T}$ for the various metastable configurations found in the simulation of the Lomer dislocation containing the vacancies. For configurations of Figures $1 \mathrm{a}$ and $\mathrm{b}$, having vacancies in adjacent sites and separated by 6 lattice spacings along the core, respectively, the absolute total potential energy difference from MD is approximately $U_{D F T}-U_{M D}=0.9 \mathrm{eV}$, or 0.0015 $\mathrm{eV}$ per atom. The error $U_{D F T}-U_{M D}$ for core sites that had the largest error $(0.03 \mathrm{eV})$ in the Lomer core with no vacancies present were reduced to 0.01 to $0.015 \mathrm{eV}$ in these configurations, while the surrounding atoms had errors similars to the no vacancy case $(-0.01$ to $0.019 \mathrm{eV}$ per atom). For the configuration of Figure 1c, with a vacancy-interstitial pair, $U_{D F T}-U_{M D}$ is approximately $0.40 \mathrm{eV}$, or $0.0007 \mathrm{eV}$ per atom. Again, the atoms which had the highest error in the Lomer core without vacancies were reduced to errors between -0.003 and 0.015 , while atoms in the center of the core have an error ranging from 0.016 to $0.03 \mathrm{eV}$ per atom. Table I summarizes the errors for each atomic configuration or density discussed above. The small range of errors obtained using the $U_{D F T}$ functional and the quenched peak assumption demonstrates that the neglect of multi-body correlations and vibrational anharmonicity at $\mathrm{T}=300 \mathrm{~K}$ are quite small, even though aharmonicity is greater in the dislocation core than in the bulk crystal.

We now investigate the potential energy of the timeaveraged "PFC" configuration of $\rho^{(1)}$. The average total potential energy including all sampled metastable states as calculated from the accelerated MD is $-23981.33 \mathrm{eV}$. The potential energy computed using Eq. 15 for the quenched PFC density, with only self-exclusion and no further correlations $\left(g^{(2)}\left(\boldsymbol{r}_{\boldsymbol{i}}, \boldsymbol{r}_{\boldsymbol{j}}\right)=1-\delta_{i j}\right)$, and adding the thermal harmonic contribution is $-23928.00 \mathrm{eV}$, which is $53.33 \mathrm{eV}$ greater than the true value. This energy difference is concentrated in the 46 atom sites along the dislocation core with vacancies, so the typical error is $\sim 1.1 \mathrm{eV}$ per core atom. This is approximately $100 \times$ larger than the typical errors associated with the functional when applied to the distinct individual configurations. Figure 3a shows the potential energy of an atom at each density peak using equation 15 and normalized by $\rho_{i}^{q}$ to obtain a per-atom value, and this energy ranges from -3.37 to $0.36 \mathrm{eV}$. In contrast, the potential energy of each atom in the actual metastable configuration shown in Figure 1a ranges only from -3.2 to $-3.4 \mathrm{eV}$ per atom, with the bulk crystal value of $-3.36 \mathrm{eV}$ per atom ${ }^{19}$ ). These energies are computed using $U_{D F T}$, but since the form of $U_{D F T}$ is identical to that of the standard PFC functional of Eq. 6, similar energies would be computed using the PFC functional, aside from an overall constant shift. Since the extreme local variations are independent of any constant shift, we conclude that use of the PFC functional could not yield a correct core structure. In other words, $\rho^{(1)}$ must be, according to DFT theory, the outcome of a true DFT functional but the PFC functionals of the form of Eq. 6 could not be accurate since they 
This is a post-print of the following article: Baker, K. L. \& Curtin, W. A. Assessment of phase-field-crystal concepts using long-time molecular dynamics. Physical Review B 91, (2015). The final publication is available at http://dx.doi.org/10.1103/PhysRevB.91.014103 @American Physical Society

TABLE I: The total average potential energy $U_{M D}$ computed in MD and using $U_{D F T}$ of equation 15 (with

harmonic vibrations added), for different distinct atomic configurations including some of the metastable states and for the PFC density corresponding to a time average of all the metastable states. All values are in unites of eV. $\Delta U=U_{D F T}-U_{M D}$ is the absolute difference between the two calculations, and the difference per atom is shown in parentheses.

\begin{tabular}{|c|c|c|c|}
\cline { 2 - 4 } \multicolumn{1}{c|}{} & $\mathrm{MD}$ & $\begin{array}{c}\text { Eq. } 15 \\
+3 / 2 N k_{B} T\end{array}$ & $\begin{array}{c}\Delta U \text { total } \\
\text { (per Atom) }\end{array}$ \\
\hline Perfect Crystal & -23442.75 & -23441.95 & $\begin{array}{l}0.80 \\
(0.0012)\end{array}$ \\
\hline $\begin{array}{c}\text { Lomer Dislocation } \\
\text { without Vacancies }\end{array}$ & -23988.21 & -23987.05 & $\begin{array}{l}1.15 \\
(0.0019)\end{array}$ \\
\hline $\begin{array}{c}\text { Two Vacancies } \\
\text { Separated }\end{array}$ & -23981.56 & -23980.67 & $\begin{array}{l}0.89 \\
(0.0015)\end{array}$ \\
\hline $\begin{array}{c}\text { Two Vacancies } \\
\text { Together }\end{array}$ & -23981.60 & -23980.68 & $\begin{array}{l}0.92 \\
(0.0015)\end{array}$ \\
\hline $\begin{array}{c}\text { Vacancy- } \\
\text { Interstitial Pair }\end{array}$ & -23980.01 & -23980.41 & $\begin{array}{l}0.40 \\
(0.0007)\end{array}$ \\
\hline $\begin{array}{c}\text { PFC } \\
\text { (Time Average of } \\
\text { Metastable States) }\end{array}$ & -23981.33 & -23928.00 & $\begin{array}{l}53.33 \\
(0.0874)\end{array}$ \\
\hline $\begin{array}{c}\text { PFC } \\
\text { (Time Average of } \\
\begin{array}{c}\text { Metastable States } \\
\text { with Hard Sphere } \\
\text { Exclusion) }\end{array}\end{array}$ & -23981.33 & -23947.33 & $\begin{array}{l}34.01 \\
(0.0558)\end{array}$ \\
\hline
\end{tabular}

predict huge errors in energies for the true structure. Any PFC functional will give some defect structure and defect energy, as a consequence of the minimization of the total free energy functional with respect to $\rho^{(1)}$ for the specified functional, but one can have little confidence that the predicted structure and energy would bear resemblance to the true values.

The extreme difference in both total and local potential energies for the "PFC" configuration is a direct consequence of calculating the interaction energies between quenched peaks that are much closer than physically possible and that do not arise simultaneously in any one actual atomistic configuration. In particular, as indicated in Figure 2a, the partially-occupied sites $\left(\rho_{i}^{q}<1.0\right)$ in the dislocation core are separated by distances ranging from 0.72 to $1.99 \AA$, far below the atomic spacing in the crystalline lattice $(2.85 \AA)$. One egregious error lies in the existence of the two very closely-spaced density peaks labelled $d$ and $e$ in Figure 2a, with $\rho_{i}=0.92$ and 0.08 and separated by $r_{d e}=0.72 \AA$, that together represent the same underlying atom but at two slightly different positions at different times. Thus, calculation

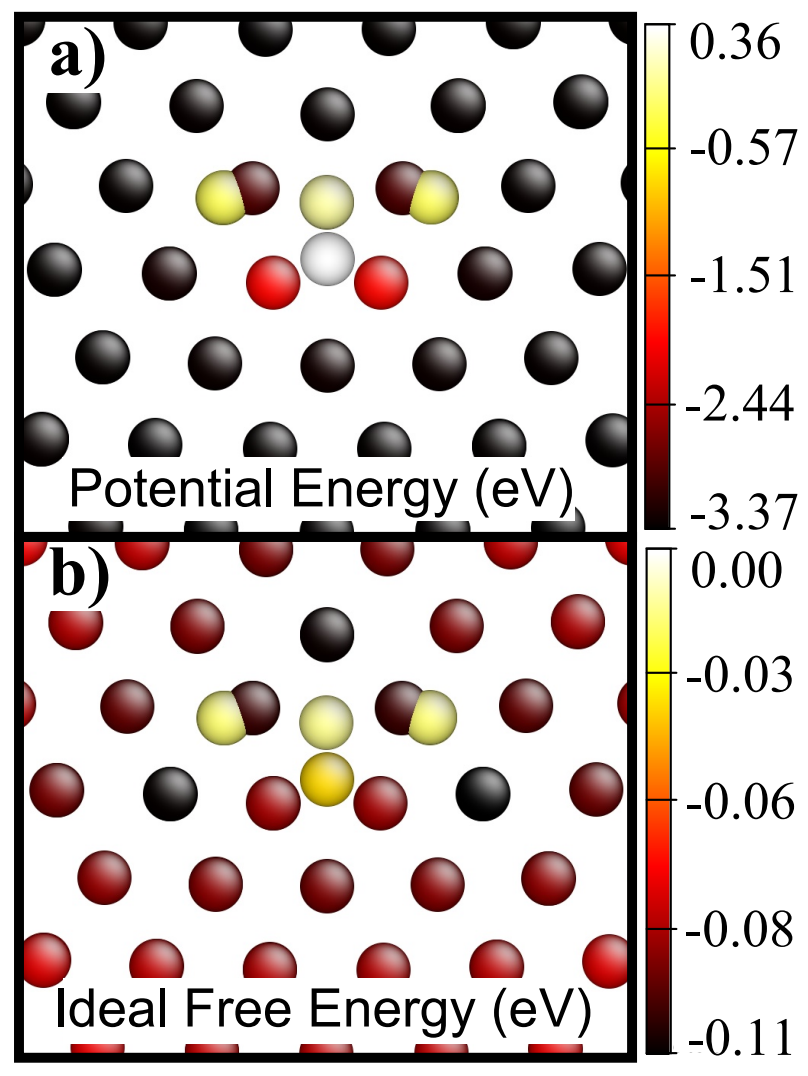

FIG. 3: (Color online) Cross section of the quenched one-particle density showing the dislocation core, with the dislocation line perpendicular to the page. Each quenched peak is colored by the a) potential energy of an atom located at each peak site computed with $U_{D F T}$ [equation 15 and $g^{(2)}\left(\boldsymbol{r}_{\boldsymbol{i}}, \boldsymbol{r}_{\boldsymbol{j}}\right)=1-\delta_{i j}$ ]; b) the ideal free energy per atom computed using equation 3 with the full $\rho^{(1)}$.

of the energy contributions from these peaks without any correlations induces self-interactions, i.e. the same atom contributing to both peaks interacts with itself leading to large errors in the energy. We can eliminate the obvious self-interactions by including a hard-sphere pair correlation function, which sets the interaction between two peaks within the specified hard-sphere diameter, $d_{\text {min }}$, to zero. This type of correlation function only accounts for some of the spatial correlations and no temporal correlations. We use a Heaviside step function, $g^{(2)}\left(\boldsymbol{r}_{\boldsymbol{i}}, \boldsymbol{r}_{\boldsymbol{j}}\right)=H\left(\left|\boldsymbol{r}_{\boldsymbol{i}}-\boldsymbol{r}_{\boldsymbol{j}}\right|-d_{\text {min }}\right)$, to create a hardcore exclusion for density peaks separated by less than $d_{\text {min }}$. For $0.73 \leq d_{\min } \leq 1.40$, the spurious interactions between peaks $d$ and $e$ are eliminated but the average potential energy is only reduced to $-23947.33 \mathrm{eV}$ which remains $34 \mathrm{eV}$ larger than the true $\mathrm{MD}$ energies. Increasing $d_{\min }$ beyond $1.41 \AA$ excludes some further unphysical interactions between densities at sites $a$ and $b$ at distance $r_{a b}=1.41 \AA$, but causes other problems. Specifically, when the energy contribution of the density at 
site $a$ is calculated, a density greater than unity is excluded corresponding to the two symmetric $b$ sites each with densities of $\rho_{b}=0.78$. This violates the sum rule $\sum_{j \neq i}^{N_{p}} \rho_{j} g^{(2)}\left(\boldsymbol{r}_{\boldsymbol{i}}, \boldsymbol{r}_{\boldsymbol{j}}\right)=N-1$ that applies to real atomistic systems. Thus, the hard sphere exclusion removes some atomic density that should be included, leading again to unphysical results. The removal of unphysical density is compounded further when $d_{\min }$ is increased beyond the peak separations $r_{c d}$ and $r_{a c}$, both of which are still well below the bulk atomic spacing of $2.85 \AA$. Therefore, implementing the same hard-sphere exclusion for all atomic sites cannot rectify the large errors in potential energy computed using the one-particle density.

As remarked earlier, the true two-body correlation function is inherently a function of space near the defect so there will always be some error when using a single correlation function $c_{L}^{(2)}$ everywhere in the sample. In particular, the sum rule will always be violated when using a single two-particle correlation function to calculate the energy of a defect from the true one-particle density. To help demonstrate this, Figure 4a compares the radial distribution functions measured from atoms located in different atomic sites in a perfect Lomer dislocation core (without vacancies) at $300 \mathrm{~K}$. At atomic site c, just outside of the dislocation core, the radial distribution function is quite similar to the bulk crystal but shifted slightly by the strain field of the dislocation around site c. At sites a and b within the core, however, the probability of finding an atom becomes more diffuse and there is significant shifting from the bulk lattice spacing. We can also compute the coordination number as a function of distance by integrating the radial distribution function. Figure $4 \mathrm{~b}$ shows the coordination number versus distance for atoms a, b, and c and for bulk atoms in Figure 4a. For site $b$, the number of nearest neighbors (distances less than $3.5 \AA$ ) is 11 while the value is 12 for the other atomic sites. Therefore, using equation 15 with a correlation function based on the bulk material will lead to an energy for site b assuming 12 neighbor atoms instead of 11. Therefore, the mismatch in the correlation function between the defect and the bulk crystal inevitably leads to an incorrect energy for atoms in the defect.

A PFC structure is obtained by minimizing the competing ideal and excess free energies. We have earlier shown that the excess free energy scales similarly to the potential energy, with an additional constant term, and just above have shown that the potential energy from the PFC structure is grossly in error. It might be argued that the overall PFC model is corrected by a compensation in the ideal free energy. Thus, we now examine the ideal free energy contributions in the true PFC density. The total and per-peak ideal Helmholtz free energy are calculated using equation 3 on the un-quenched $\rho^{(1)}$ density generated from accelerated MD (Figure 3b). The total ideal free energy is $-53.40 \mathrm{eV}$, with an average value of -0.09 $\mathrm{eV}$ per atom $\left(\sim-3.5 k_{B} \mathrm{~T}\right)$. The individual per-peak values of the potential energy (used in equation 15) and $F^{i d}$ are plotted in Figure 3a and b, respectively. The varia-
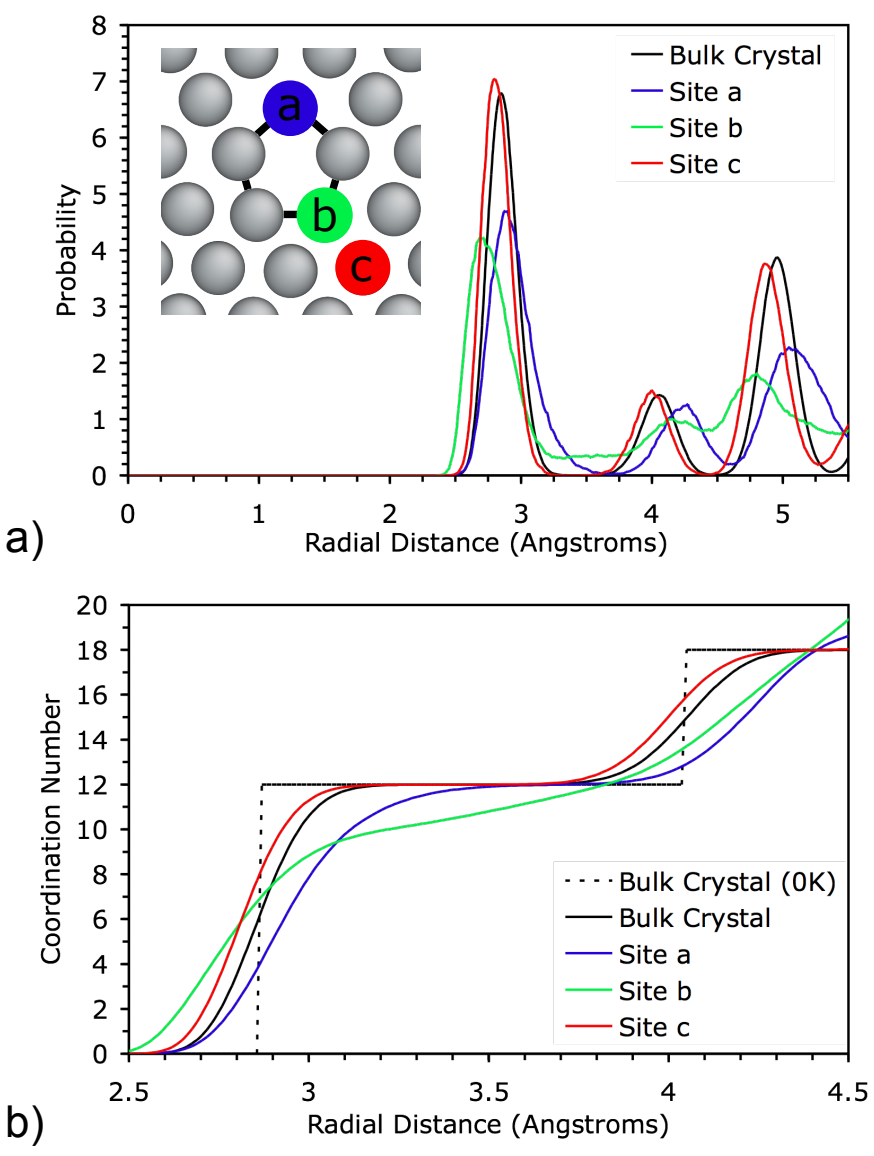

FIG. 4: (Color online) a) The radial distribution function measured using MD at $300 \mathrm{~K}$ of the bulk crystal and several atoms located in or near a Lomer dislocation core (with no vacancies present in the core). The inset indicates the location of the reference atoms in or near the dislocation core. For easy identification, the core atoms are connected by a solid black lines. b) The coordination number of the same set of atoms. The 0K coordination number is included as a reference.

tions in the ideal free energy contribution from each PFC density peak are generally very low, ranging from zero to $-0.11 \mathrm{eV}$, as compared to the magnitude and variations in the potential energy contributions. These values are similar to those obtained for individual configurations; for the metastable configuration in Figure 1a has $F^{i d}=$ $-47.88 \mathrm{eV}$, or roughly $-0.08 \mathrm{eV}$ per atom $\left(\sim-3.1 k_{B} T\right)$ and ranging from -0.06 to $-0.11 \mathrm{eV}$ per atom. Since the ideal entropy in the liquid is $1.5 k_{B} T$, it is not possible to compensate for the large errors in potential energy $(\sim 1$ $\mathrm{eV}$ /atom) through differences in the ideal entropy. As noted above, while the overall constant in Eq. 6 could correct on average for the large discrepancy in potential energy, such a constant shift is unable to account for the large energy differences among different atomic sites in the core of the defect. 
This is a post-print of the following article: Baker, K. L. \& Curtin, W. A. Assessment of phase-field-crystal concepts using long-time molecular dynamics. Physical Review B 91, (2015). The final publication is available at http://dx.doi.org/10.1103/PhysRevB.91.014103 @American Physical Society

\section{Kinetics of Transitions between Configurations}

A key aspect of PFC models is the dynamic evolution in time over "diffusive" time scales. The question is whether such a time evolution is realistic or accurate. PFC only operates on the evolution of $\rho^{(1)}$ and only uses one (simplified) correlation function. This means, as noted above for metastable states, that it is not possible to identify any particular atomic transition state configuration. Therefore, reaction rates between metastable states cannot be calculated with high accuracy, and any state-to-state dynamics cannot be captured. Thus, using equation 11 or 12 is numerically efficient but lacks a connection to the controlling features of atomistic dynamics in solid systems. In addition, the dynamic equations used by PFC are deterministic and only move downhill in the free energy; it is thus possible to reach and become stuck in a metastable energy minimum. Only by adding an artificial random noise term can the system move between metastable states.

While one could envision tuning the PFC evolution to a match a particular transition rate, the PFC model uses just one constant mobility factor in Eqs. 11 and 12 . Using the Nudged Elastic Band method ${ }^{60}$, we have computed the saddle states of the potential energy surface between the various metastable energy minima associated with the various configurations visited during the MD simulation. The energy barriers for the observed transitions for vacancy motions span a wide range from $0.29 \mathrm{eV}$ to $0.74 \mathrm{eV}$, while the bulk vacancy migration energy is $0.61 \mathrm{eV}$. Therefore, using a single mobility coefficient can not distinguish between the hugely different actual transition rates that occur naturally in the system. In our specific example, we can imagine starting the system in one configuration and asking for the timeaveraged evolution of $\rho^{(1)}$ as the system evolves toward the final time-averaged configuration. The PFC model could not capture the dynamics associated with transitions ranging over almost $0.5 \mathrm{eV}$ in barrier height. This further implies that the computed $\rho^{(1)}$ at any instant in time cannot be considered as any true realization of the evolving one-particle density.

\section{CONCLUSIONS}

In summary, we have generated an essentially exact equilibrium one-particle density function $\rho^{(1)}$ using accelerated MD for a non-trivial defect structure in a crystalline material. From this result, we argue that any type of PFC model that relies on the one-particle density and standard free-energy functionals that do not include accurate multi-particle correlations cannot quantitatively predict atomic structures, energies, or kinetics. This conclusion goes beyond the actual operational limitations of most current PFC implementations, which use low-resolution density profiles that do not correspond to the sharp peaks associated with thermal vibrations in crystalline systems. Without knowledge of the manybody correlation function, it is impossible to distinguish the individual atomic configurations that contribute to the one-particle density, or to compute the energy of the system or the kinetic events occurring as the system samples the available phase space. We have investigated the reliability of an alternative functional based on the potential energy, and we have demonstrated that this functional can accurately describe the energetics of any one distinct configuration found in the MD. Thus, for equilibrium properties in solids at low and moderate temperatures, this functional is strongly preferable to current PFC functionals. There is no solution to overcome the limitation of the PFC time evolution, which has no formal basis nor any connection to accepted transition state theory. Instead, using a combination of calculating the free energy of metastable states with $\mathrm{PFC}$, along with a standard transition-state-based description of evolution using a master-equation and/or kinetic Monte-Carlo type approach is one possible avenue for pursuing time evolution, but such methods do not have the computational efficiency of a standard PFC model.

In general, the PFC model is an interesting and convenient framework for studying the evolution of simple systems where discrete atomic-scale phenomena play a key role in the system evolution. PFC has been used to model complex defect structures and kinetics, like dislocation dynamics $^{23,24,30-32}$, structural phase changes $^{44}$, grain boundary energies and evolution ${ }^{8,26,27,33-35}$, and vacancy diffusion $^{61}$. In some cases, the results for some complex problems show qualitative trends consistent with basic models and direct molecular simulations ${ }^{26,27,62}$. In addition, classical DFT methods using advanced functionals and highly-spatially-resolved density profiles can provide accurate thermodynamics in some problems, such as the solid-liquid interface and its fluctuations ${ }^{63-65}$ where atomic fluctuations occur on fast time scales. However, based on our results for a typical problem arising in materials science, the PFC model does not appear to be suitable for quantitative computation of equilibrium defect structures in crystalline systems, such as dislocation cores, grain boundaries, or crack tips, or their microscopic evolution. For these classes of problems, the more-computationally-intensive methods such as accelerated MD appear to remain necessary to capture details at the level required for quantitative prediction in materials science.

\section{ACKNOWLEDGEMENTS}

The authors would like to thank Art Voter for useful discussions. Support for this work was partially provided through a European Research Council Advanced Grant, "Predictive Computational Metallurgy", ERC Grant agreement No. 339081 - PreCoMet and partially funded by EPFL through its support for the Laboratory for Multiscale Mechanics Modelling (LAMMM). 
This is a post-print of the following article: Baker, K. L. \& Curtin, W. A. Assessment of phase-field-crystal concepts using long-time molecular dynamics. Physical Review B 91, (2015). The final publication is available at http://dx.doi.org/10.1103/PhysRevB.91.014103 @American Physical Society

1 A. F. Voter, Journal of Chemical Physics 106, 4665 (1997).

2 A. F. Voter, Physical Review Letters 78, 3908 (1997).

3 A. F. Voter, Physical Review B 57, R13985 (1998).

4 M. R. Sørensen and A. F. Voter, Journal of Chemical Physics 112, 9599 (2000).

${ }^{5}$ D. T. Gillespie, Journal of Computational Physics 22, 403 (1976).

6 D. T. Gillespie, The Journal of Physical Chemistry 81, 2340 (1977).

7 D. T. Gillespie, Journal of Computational Physics 28, 395 (1978).

8 K. Elder, M. Katakowski, M. Haataja, and M. Grant, Physical Review Letters 88, 245701 (2002).

9 T. Ramakrishnan and M. Yussouff, Physical Review B 19, 2775 (1979).

10 A. Haymet and D. W. Oxtoby, Journal of Chemical Physics 74, 2559 (1981).

11 B. Devincre and L. Kubin, Materials Science and Engineering: A 234, 8 (1997).

12 J. P. Hirth and J. Lothe, Theory of Dislocations, 2nd ed. (John Wiley \& Sons, Inc., 1982).

13 D. L. Olmsted, L. G. H. Jr., W. Curtin, and R. Clifton, Modelling Simul. Mater. Sci. Eng. 13, 371 (2005).

14 L. G. H. J. Gerard Paul M. Leyson, William A. Curtin and C. F. Woodward, Nature Materials 9, 750 (2010).

15 V. B. Shenoy, R. Miller, E. Tadmor, R. Phillips, and M. Ortiz, Physical Review Letters 80, 742 (1998).

16 V. Bulatov, F. F. Abraham, L. Kubin, B. Devincre, and S. Yip, Letters to Nature 391, 669 (1998).

17 M. S. Daw and M. Baskes, Physical Review Letters 50, 1285 (1983).

18 M. S. Daw and M. Baskes, Physical Review B 29, 6443 (1984).

19 F. Ercolessi and J. B. Adams, Europhysics Letters 26, 583 (1994).

20 M. J. Mills, M. S. Daw, and S. M. Foiles, Ultramicroscopy 56, 79 (1994).

21 Y. Mishin, D. Farkas, M. Mehl, and D. Papaconstantopoulos, Physical Review B 59, 3393 (1999).

${ }^{22}$ X.-Y. Lui, F. Ercolessi, and J. B. Adams, Modelling Simul. Mater. Sci. Eng. 12, 665 (2004).

23 J. Berry, N. Provatas, J. Rottler, and C. Sinclair, Physical Review B 86, 224112 (2012).

24 J. Berry, N. Provatas, J. Rottler, and C. Sinclair, Physical Review B 89, 214117 (2014).

25 P. Y. Chan, N. Goldenfeld, and J. Dantzig, Physical Review E 79, 035701 (2009).

26 A. Jaatinen, C. Achim, K. Elder, and T. Ala-Nissila, Physical Review E 80, 031602 (2009).

27 A. Jaatinen, C. Achim, K. Elder, and T. Ala-Nissila, Technische Mechanik 30, 169 (2010).

28 J. P. Hansen and I. R. McDonald, Theory of simple liquids, 3rd ed. (Elsevier, 2006).

29 R. Evans, in Lectures at 3rd Warsaw School of Statistical Physics (2009).

30 P. Stefanovic, M. Haataja, and N. Provatas, Physical Review Letters 96, 225504 (2006).

31 P. Y. Chan, G. Tsekenis, J. Dantzig, K. A. Dahmen, and N. Goldenfeld, Physical Review Letters 105, 015502 (2010).
32 P. Stefanovic, M. Haataja, and N. Provatas, Physical Review E 80, 046107 (2009).

33 D. L. Olmsted, D. Buta, A. Adland, S. M. Foiles, M. Asta, and A. Karma, Physical Review Letters 106, 046101 (2011).

34 J. Berry, K. Elder, and M. Grant, Physical Review B 77, 224114 (2008).

35 J. Mellenthin, A. Karma, and M. Plapp, Physical Review B 78, 184110 (2008).

36 K. Elder, N. Provatas, J. Berry, P. Stefanovic, and M. Grant, Physical Review B 75, 064107 (2007).

37 S. van Teeffelen, R. Backofen, A. Voigt, and H. Löwen, Physical Review E 79, 051404 (2009).

38 A. Jaatinen and T. Ala-Nissila, Physical Review E 82, 061602 (2010).

39 R. Backofen and A. Voigt, Journal of Physics: Condensed Matter 22, 364104 (2010).

40 G. I. Tóth, G. Tegze, T. Pusztai, G. Tóth, and L. Gránásy, Journal of Physics: Condensed Matter 22, 364101 (2010).

41 N. Pisutha-Arnond, V. Chan, M. Iyer, V. Gavini, and K. Thornton, Physical Review E 87, 013313 (2013).

42 D. Stroud and N. W. Ashcroft, Physical Review B 5, 371 (1972).

43 G. Caginalp, Physical Review A 39, 5887 (1989).

44 M. Greenwood, N. Provatas, and J. Rottler, Physical Review Letters 105, 045702 (2010).

45 M. Greenwood, N. Ofori-Opoku, J. Rottler, and N. Provatas, Physical Review B 84, 064104 (2011).

46 L. Gránásy, G. Tegze, G. I. Tóth, and T. Pusztai, Philosophical Magazine 91, 123 (2011).

47 M. Oettel, S. Dorosz, M. Berghoff, B. Nestler, and T. Schilling, Physical Review E 86, 021404 (2012).

48 A. D. J. Haymet, in Fundamentals of Inhomogeneous Flu$i d s$, edited by D. Henderson (Mercel Dekker, Inc., 1992) Chap. 9, pp. 363-405.

49 W. Curtin, Journal of Chemical Physics 88, 7050 (1988).

50 R. LeSar, R. Najafabadi, and D. J. Srolovitz, Physical Review Letters 63, 624 (1989).

${ }^{51}$ G. K.-L. Chan and R. Finken, Physical Review Letters 94, 183001 (2005).

52 H. Emmerich, H. Löwen, R. Wittkowski, T. Gruhn, G. I. Tóth, G. Tegze, and L. Gránásy, Advances in Physics 61, 665 (2012).

53 S. J. Plimpton, Journal of Computational Physics 117, 1 (1995).

54 D. L. Olmsted, R. Phillips, and W. A. Curtin, Modelling Simul. Mater. Sci. Eng. 12, 1 (2004).

55 D. Perez, B. P. Uberuaga, Y. Shim, J. G. Amar, and A. F. Voter, in Annual Reports in Computational Chemistry, Vol. 5 (Elsevier, 2009) Chap. 4, pp. 79-98.

${ }^{56}$ R. LeSar, R. Najafabadi, and D. J. Srolovitz, J. Chem. Phys. 94, 5090 (1991).

57 J. Li, S. Sarkar, W. T. Cox, T. J. Lenosky, E. Bitzek, and Y. Wang, Physical Review B 84, 054103 (2011).

58 S. Sarkar, J. Li, W. T. Cox, E. Bitzek, T. J. Lenosky, and Y. Wang, Physical Review B 86, 014115 (2012).

59 The full potential energy can be computed using $\rho^{(1)}(\boldsymbol{r})$ by integrating the density bins instead of using the quenched density $\rho_{q}^{(1)}(\boldsymbol{r})$. However, this calculation is very sensitive to the bin size and the distribution of the one-particle den- 
This is a post-print of the following article: Baker, K. L. \& Curtin, W. A. Assessment of phase-field-crystal concepts using long-time molecular dynamics. Physical Review B 91, (2015). The final publication is available at http://dx.doi.org/10.1103/PhysRevB.91.014103 @American

sity (and therefore the sampling time in accelerated MD). Attempts to obtain the full potential energy by integrating the density produce errors that are at least an order of magnitude larger than using the quenched density plus the harmonic approximation for the thermal contribution.

${ }^{60}$ H. Jónsson, G. Mills, and K. W. Jacobsen, "Classical and quantum dynamics in condensed phase simulations," (World Scientific, 1998) Chap. Nudged elastic band method for finding minimum energy paths of transitions, pp. 385-404.
61 S. van Teeffelen, C. V. Achim, and H. Lówen, Physical Review E 87, 022306 (2013).

62 S. Muralidharan and M. Haataja, Physical Review Letters 105, 126101 (2010).

63 R. Ohnesorge, H. Löwen, and H. Wagner, Physical Review A 43, 2870 (1991).

${ }^{64}$ R. Ohnesorge, H. Löwen, and H. Wagner, Europhysics Letters 22, 245 (1993).

65 R. Ohnesorge, H. Löwen, and H. Wagner, Physical Review E 50, 4801 (1994). 